AEI-2000-3

BONN-TH-2000-02

IASSNS-HEP-00/21

hep-th/0003110

\title{
On relevant boundary perturbations of unitary minimal models
}

\author{
A. Recknage ${ }^{1}$, D. Roggenkamp ${ }^{2}$ and V. Schomerus ${ }^{3,4}$ \\ ${ }^{1}$ Max-Planck-Institut für Gravitationsphysik \\ Albert-Einstein-Institut \\ Am Mühlenberg 1, D-14476 Golm, Germany \\ 2 Physikalisches Institut der Universität Bonn \\ Nußallee 12, D-53115 Bonn, Germany \\ ${ }^{3}$ II. Institut für Theoretische Physik, Universität Hamburg, \\ Luruper Chaussee 149, D-22761 Hamburg, Germany \\ 4 Institute for Advanced Study, School of Natural Sciences, \\ Olden Lane, Princeton, NY 08540, U.S.A.
}

\begin{abstract}
We consider unitary Virasoro minimal models on the disk with Cardy boundary conditions and discuss deformations by certain relevant boundary operators, analogous to tachyon condensation in string theory. Concentrating on the least relevant boundary field, we can perform a perturbative analysis of renormalization group fixed points. We find that the systems always flow towards stable fixed points which admit no further (non-trivial) relevant perturbations. The new conformal boundary conditions are in general given by superpositions of 'pure' Cardy boundary conditions.
\end{abstract}

e-mail addresses: anderl@aei-potsdam.mpg.de, roggenka@th.physik.uni-bonn.de, vschomer@x4u2.desy.de 


\section{Introduction}

Conformal field theory on surfaces with boundaries, or boundary CFT for short, has recently attracted new interest because it provides the framework for a world-sheet analysis of D-branes in string theory. It has also applications to various systems of condensed matter physics such as the three-dimensional Kondo effect [1], fractional quantum Hall fluids (see e.g. [2]) and other quantum impurity problems.

A lot of progress has been made in clarifying the rather rich intrinsic structure of boundary CFTs, but deformations away from the conformal point have been investigated less systematically in the literature. Their study should provide some insight into the structure of the space of boundary theories and its renormalization group fixed points, besides possible applications in condensed matter physics. Such deformations are also important in the string theory context, where flows triggered by a relevant boundary operator appear as tachyon condensation and may result e.g. in the formation of (non-BPS) bound states of branes, see $[3,4]$ and the more recent string field theory considerations in $[5,6]$.

Most of the existing literature on relevant boundary deformations rests on the thermodynamic Bethe ansatz (TBA), on scattering matrices or on the truncated conformal space approach (TCSA), see e.g. [7,8]. These methods can provide (non-perturbative) information about RG flows even between the fixed points, but they do not lend themselves easily to model independent investigations. Some general aspects of conformal perturbation theory have been discussed in the work of Affleck and Ludwig [1,9], leading to the formulation of the so-called "g-theorem". Here, we want to use and refine these techniques to study specific relevant boundary deformations of arbitrary unitary Virasoro minimal models. The problem we attack can be viewed as a boundary analogue of Zamolodchikov's analysis of relevant bulk perturbations of minimal models [10] which showed that deformation of the minimal model $M_{m}$ by the least relevant bulk field $\varphi_{(1,3)}$ induces an RG flow to a new fixed point corresponding to the model $M_{m-1}$.

In order to identify the CFT at the new fixed point, Zamolodchikov simply checks that, after the deformation, the central charge agrees with $c_{m-1}$ up to higher order corrections in the coupling constant $\lambda$. It is a (technically important) peculiarity of the least relevant field $\varphi_{(1,3)}$ that the expansion in $\lambda$ coincides with an expansion in the parameter $1 / \mathrm{m}$.

In the study of boundary perturbations, the quantity of interest is the ground-state degeneracy $g_{a}=\langle\mathbf{1}\rangle_{a}$, i.e. the vacuum expectation value of the identity in the presence of the boundary condition $a$, which also plays the main role in the g-theorem. In close analogy to [10], we first determine the new fixed point from the beta function - to low order in the coupling constant or, at the same time, to low order in $1 / \mathrm{m}$. (The same "peculiarity" as in the bulk case can be exploited for the least relevant boundary operator $\psi_{(1,3)}$.) Then we calculate the change of $g_{a}$ in an expansion in $1 / \mathrm{m}$. Since the set of possible boundary conditions for Virasoro minimal models is discrete, the first few terms suffice to identify the boundary condition which is reached at the perturbative fixed point of the $\psi_{(1,3)}$-flow

- up to some residual ambiguities which can be resolved by studying one-point functions of other primary bulk fields.

In the next section, we recall some facts about minimal models on the disk, postponing some lengthier formulas to the Appendix. The perturbative analysis of relevant boundary deformations is carried out in Section 3, while Section 4 contains conclusions and remarks on possible generalizations. 
On a qualitative level, there are two main lessons to be drawn from the results of Section 3. First of all, the RG flows generically end up with conformal boundary conditions that can only be described by superpositions of Cardy boundary states. It appears, therefore, that one cannot attribute a more fundamental meaning to such "pure" boundary conditions (with a unique vacuum state in the spectrum) than to superpositions thereof. Both types occur on an equal footing in the space of conformal boundary conditions associated with a given CFT on the Riemann sphere. Similar effects show up in string theory, where single D-branes can be continuously deformed into systems of several branes, see $[11,4]$. The physical meaning of these "systems" in terms of two-dimensional phase diagrams can, however, be very different: In the recent work [12], Affleck has connected superpositions of boundary conditions with long range order of boundary spins, and thus to first order phase transitions. While [12] focuses on the tri-critical Ising model $(m=4)$, this interpretation certainly generalizes to the boundary states we will meet below, simply because the main "world-sheet signal" for an ordered phase, the occurrence of additional dimension zero operators, is present in all these cases.

The second comment we would like to make is that some naive expectations about RG flows fail in the context of boundary perturbations: Renormalization can be thought of as integrating out degrees of freedom, and intuitively one would expect that the more relevant the perturbing operator is the more degrees of freedom are integrated out. In the bulk case [10], the CFT reached after perturbing a minimal model with the least relevant field does contain further relevant operators, in accordance with this simple picture. In contrast, the boundary flows found in the present paper, and in fact most examples studied in the literature so far, lead to stable fixed points (in the sense that no relevant boundary operators remain in the spectrum). On the other hand, there are counterexamples like in the three-states Potts model [13] and in SU(2) WZW models [14]. A perhaps even more counterintuitive behaviour of boundary perturbations was discovered in $[11,4]$ : In some situations, a (seemingly irreversible) relevant boundary perturbation can be "undone" by a sequence of (invertible) marginal deformations in the bulk and on the boundary. Such "failures" of the Wilsonian picture of RG flows are most probably due the the very different weights of bulk and boundary degrees of freedom, but we feel that the phenomenon deserves further study.

\section{Boundary conditions for minimal models}

We consider unitary Virasoro minimal models $M_{m}$ with central charges $c_{m}=1-\frac{6}{m(m+1)}$ for $m=3,4, \ldots$, and with diagonal modular invariant partition functions. The left- and right-moving conformal dimensions of the primaries $\varphi_{i}(\zeta, \bar{\zeta})$ are given by the Kac table

$$
h_{m}(r, s)=\frac{((m+1) r-m s)^{2}-1}{4 m(m+1)}
$$

with $i \equiv(r, s) \in I_{\mathrm{Kac}}:=\{(\bar{r}, \bar{s}) \mid 1 \leq \bar{r} \leq m-1,1 \leq \bar{s} \leq m\} / \sim$, where " " denotes the conformal grid symmetry $(r, s) \sim(m-r, m+1-s)$. Conformal boundary conditions on such a "bulk CFT" can be described by boundary states

$$
\left.\| a\rangle\rangle=\sum_{i \in I_{\mathrm{Kac}}} B_{a}^{i}|i\rangle\right\rangle .
$$


To each irreducible representation of the Virasoro algebra, there is an associated Ishibashi state $|i\rangle\rangle=|r, s\rangle\rangle$ satisfying linear conditions $\left.\left(L_{n}-\bar{L}_{-n}\right)|i\rangle\right\rangle=0$ which guarantee conformal invariance of the system on the upper half-plane or the unit disk, see $[15,16]$ for the construction. The complex coefficients $B_{a}^{i}$ are subject to non-linear equations like sewing constraints [17] and conditions from modular covariance [16]. The latter require that the quantity

$$
Z_{a b}(q):=\left\langle\left\langle b\left\|\tilde{q}^{L_{0}-\frac{c}{24}}\right\| a\right\rangle\right\rangle=\sum_{i, j} \overline{B_{b}^{i}} B_{a}^{i} S_{i j} \chi_{j}(q)=\sum_{j} n_{a b}^{j} \chi_{j}(q)
$$

can be interpreted as partition function of a CFT on a strip, with boundary conditions $a$ and $b$ imposed along the boundaries. In other words, the $n_{a b}^{j}$ must be non-negative integers. Above, we have introduced the conformal characters $\chi_{j}(q):=\operatorname{tr}_{\mathcal{H}_{j}} q^{L_{0}-\frac{c}{24}}$ of the irreducible modules $\mathcal{H}_{j}$, with $q=e^{2 \pi i \tau}$ and $\tilde{q}=e^{-2 \pi i / \tau}$, and the modular $S$-matrix from $\chi_{i}(\tilde{q})=\sum_{j} S_{i j} \chi_{j}(q)$.

For rational diagonal models, Cardy found a general solution to the constraints (3): Specializing to our case, the Cardy boundary states carry the same labels $a \in I_{\mathrm{Kac}}$ from the Kac table as the Ishibashi states, and the coefficients are

$$
B_{a}^{i}=\frac{S_{a i}}{\sqrt{S_{0 i}}}
$$

$(0=(1,1)$ denotes the vacuum representation $)$ with the minimal model $S$-matrix

$$
S_{(r, s)\left(r^{\prime}, s^{\prime}\right)}=\sqrt{\frac{8}{m(m+1)}}(-1)^{1+r s^{\prime}+s r^{\prime}} \sin \left(\pi \frac{m+1}{m} r r^{\prime}\right) \sin \left(\pi \frac{m}{m+1} s s^{\prime}\right) .
$$

The partition functions calculated with Cardy's boundary states have the simple form $Z_{a b}(q)=\sum_{j} N_{a b}^{j} \chi_{j}(q)$, where the minimal model fusion rules $N_{i j}^{k}$ can be read off from

$$
\phi_{(r, s)} \times \phi_{\left(r^{\prime}, s^{\prime}\right)}=\sum_{\substack{r^{\prime \prime}=\left|r-r^{\prime}\right|+1 \\
r+r^{\prime}+r^{\prime \prime} \text { odd }}}^{r_{\max }} \sum_{\begin{array}{l}
s^{\prime \prime}=\left|s-s^{\prime}\right|+1 \\
s+s^{\prime}+s^{\prime \prime} \text { odd }
\end{array}}^{s_{\max }} \phi_{\left(r^{\prime \prime}, s^{\prime \prime}\right)}
$$

with summations running up to $r_{\max }=\min \left(r+r^{\prime}-1,2 m-r-r^{\prime}-1\right)$ resp. $s_{\max }=$ $\min \left(s+s^{\prime}-1,2 m-s-s^{\prime}+1\right)$.

The Cardy boundary states $\| a\rangle\rangle$ form a complete set in the sense [18] that the matrix of coefficients $B_{a}^{i}$ is invertible; see also [19]. This implies that any other boundary state $\left.\left.\| A\right\rangle\right\rangle$ is a (complex) linear combination of Cardy states. If we impose the natural compatibility condition that $Z_{A 0}(q)$, where $\left.\left.\| 0\right\rangle\right)$ is the Cardy state associated with the vacuum representation, is a partition function, then $\| A\rangle\rangle$ is in fact an element of the $\mathbb{Z}_{+}$-lattice generated by the Cardy boundary states,

$$
\left.\left.\| A\rangle\rangle=\sum_{a \in I_{\mathrm{Kac}}} n_{A}^{a} \| a\right\rangle\right\rangle \quad \text { with } n_{A}^{a} \in \mathbb{Z}_{+} .
$$

The partition functions $Z_{a a}(q)$ describe the spectrum of boundary fields - of field operators which can be inserted (only) along world-sheet boundaries where the boundary condition 
$a$ is imposed (in distinction to the bulk fields $\varphi_{i}(\zeta, \bar{\zeta})$ which live in the interior of the disk). The boundary operators are in one-to-one correspondence to the space of states of the boundary CFT, for Cardy type boundary conditions given by

$$
\mathcal{H}_{a a}=\bigoplus_{j \in I_{\mathrm{Kac}}} \mathcal{H}_{j}^{\oplus N_{a a}^{j}}
$$

The $\mathcal{H}_{j}$ are irreducible representations of the Virasoro algebra, again with highest weights from the Kac table (1). Note that Cardy boundary conditions are distinguished among the $\| A\rangle\rangle$ by the property that $\mathcal{H}_{a a}$ contains the vacuum precisely once.

Partition functions $Z_{a b}(q)$ with $a \neq b$ are associated to spaces of so-called boundary condition changing operators, which induce a jump in the boundary condition. (In string theory, they correspond to excitations of open strings stretched between two different D-branes.)

The decomposition (8) in particular allows us to determine the set of relevant boundary operators: We have $h_{m}(r, s)<1$ iff $s=r, r \pm 1, r+2$, and the least relevant boundary field $\psi_{(1,3)}$ has conformal dimension

$$
h_{m}(1,3)=\frac{m-1}{m+1} .
$$

It appears in the state space of each Cardy boundary condition with the exception of the series $\| a\rangle\rangle=\|(r, 1)\rangle$, for which $\mathcal{H}_{a a}$ contains no (non-trivial) relevant boundary fields at all.

A conformal field theory is "solved" once all correlation functions are known. The $B_{a}^{i}$ determine [20] the one-point functions of primary bulk fields $\varphi_{i}(\zeta, \bar{\zeta})$ in the presence of the boundary condition $a$,

$$
\left\langle\varphi_{i}(\zeta, \bar{\zeta})\right\rangle_{a}=\frac{B_{a}^{i}}{|1-\zeta \bar{\zeta}|^{2 h_{i}}}
$$

and with the help of the structure constants in the bulk operator product expansion and of the conformal Ward identities, one can in principle compute arbitrary correlators which involve only bulk fields. Correlators of (bulk and) boundary fields can be treated in the same way once the structure constants in the bulk-boundary OPE [20] and in the OPE of boundary fields [17]

$$
\psi_{k}^{a b}\left(e^{i \theta_{1}}\right) \psi_{l}^{c d}\left(e^{i \theta_{2}}\right) \approx \sum_{m} \delta^{b, c} C_{k l m}^{a b d}\left(\theta_{1}-\theta_{2}\right)^{h_{m}-h_{k}-h_{l}} \psi_{m}^{a d}\left(e^{i \theta_{2}}\right)
$$

(for $\theta_{1} \approx \theta_{2}$ and $0 \leq \theta_{2}<\theta_{1}<2 \pi$ ) are known. All these structure constants are subject to non-linear equations arising from duality or sewing constraints [17]. For Virasoro minimal models, solutions for the constants in eq. (9) were constructed by Runkel [21]:

$$
C_{k l m}^{a b c}=\mathrm{F}_{b m}\left[\begin{array}{ll}
a & c \\
k & l
\end{array}\right]
$$

where $\mathrm{F}$ is the fusing matrix of the (chiral) Virasoro model; explicit expressions for special entries are given in the Appendix, see [21] for the remaining cases. That the boundary 
OPE is related to the fusing matrix is a more general phenomenon, as was shown recently in [22]; see also [19] for earlier investigations.

We have collected defining data of Virasoro minimal models on the unit disk. For what follows, the most important pieces of information are the one-point functions of the identity operator for Cardy boundary conditions $a=(r, s)$,

$$
g_{a} \equiv B_{a}^{0}=\left(\frac{8}{m(m+1)}\right)^{\frac{1}{4}} \frac{\sin \frac{\pi r}{m} \sin \frac{\pi s}{m+1}}{\left(\sin \frac{\pi}{m} \sin \frac{\pi}{m+1}\right)^{\frac{1}{2}}},
$$

the boundary OPE structure constants $C_{k k m}^{a a a}$ for the special case of a constant boundary condition, and the spectra of boundary (condition changing) operators contained in $\mathcal{H}_{a b}$.

\section{Relevant boundary perturbations}

Given a boundary CFT with correlation functions $\langle\cdot\rangle_{a}$, where $a$ denotes the boundary condition along the unit circle, we can select a boundary field $\psi$ and try to define a new set of correlators by the (formal) expression

$$
\begin{aligned}
\left\langle\varphi_{i_{1}}\left(\zeta_{1}, \bar{\zeta}_{1}\right) \cdots \varphi_{i_{N}}\left(\zeta_{N}, \bar{\zeta}_{N}\right)\right\rangle_{a ; \lambda \psi} & \\
& =\left\langle\varphi_{i_{1}}\left(\zeta_{1}, \bar{\zeta}_{1}\right) \cdots \varphi_{i_{N}}\left(\zeta_{N}, \bar{\zeta}_{N}\right) P \exp \left\{\lambda \varepsilon^{h_{\psi}-1} S_{\psi}\right\}\right\rangle_{a}
\end{aligned}
$$

The symbol $P$ denotes path ordering of the exponential of the perturbation

$$
S_{\psi}:=\int_{\partial \Sigma} \psi(s) d s
$$

inserted along the boundary of the world-sheet $\Sigma$ (here: a disk of radius $L$ ). Path ordering takes care of the fact that boundary fields in general are not local wrt. each other or wrt. themselves - cf. the specific ordering of world-sheet arguments and the appearance of the $\delta$-symbol for boundary condition changing operators in the expansion (9).

The higher order terms of the exponential series require UV regularization e.g. by restricting all integrals to the region $\left|x_{i}-x_{j}\right|>\varepsilon$, where $\varepsilon$ is some UV cutoff. We introduce a dimensionless renormalization group parameter $l$ that regulates the scale of the theory, $\frac{L}{\varepsilon}(l)=e^{-l}$. We will keep the disk circumference $2 \pi L$ fixed so that we need not deal with IR divergences; they could also be taken care of by introducing a finite temperature, see e.g. [1].)

The cutoff $\varepsilon$ was already used above to render the real parameter $\lambda$ in (12) dimensionless. $\lambda$ is the strength of the perturbation in direction of $\psi$. If the conformal dimension of $\psi$ is 1 , i.e. if $\psi$ is marginal, then the new set of correlation functions may define a new boundary conformal field theory. A criterion which ensures that such a $\psi$ yields a truly marginal deformation to all orders in $\lambda$ was established in [11], where also various general results on the structure of the new CFT were obtained, providing some insight into the moduli space of conformal boundary conditions (or of D-branes).

Here, we are interested in deformations with $h_{\psi}<1$. Such relevant perturbations introduce dimensionful quantities into the theory and therefore break conformal invariance. The 
latter is restored only at fixed points of the renormalization group flow triggered by $\psi$, and we reach a new conformal boundary condition for the same bulk CFT that we started from. (Perturbations by boundary operators do not affect the local properties in the interior of the disk, thus the "parent" bulk theory is not changed.)

Several approaches can be applied to exhibit the properties of the RG flow and of the new fixed points, e.g. TBA and TSCA methods. In this paper, we use (zero temperature) conformal perturbation theory (see [23] for a nice introduction to the bulk case), which is suitable to study a small neighbourhood of the original theory $\lambda=0$.

In this approach, new fixed points are determined from the zeroes of the beta function $\beta(\lambda)=d \lambda / d \ln (L / \varepsilon)=d \lambda / d l$, which in turn is computed perturbatively (in $\lambda$ ) from the OPE of the field $\psi$, see e.g. $[9,1,23]$,

$$
\beta(\lambda)=\left(1-h_{\psi}\right) \lambda+C_{\psi \psi \psi}^{a a a} \lambda^{2}+O\left(\lambda^{3}\right)
$$

Note that $\lambda$ denotes the renormalized coupling constant here. We have assumed that no other non-trivial relevant operators appear in the OPE of $\psi$ with itself, as is the case for the field $\psi=\psi_{(1,3)}$ we are going to study; otherwise, new counterterms arise which introduce additional coupling constants and render the analysis much more complicated; see [23]. To this lowest non-trivial order, $\beta\left(\lambda^{*}\right)=0$ yields a fixed point at [9]

$$
\lambda^{*}=-\frac{y}{C_{\psi \psi \psi}^{a a a}} \quad \text { with } \quad y:=1-h_{\psi}
$$

This value can be inserted into the perturbative expansion of the correlators (12), and we can in particular determine the one-point function of the identity $g_{a}(\lambda) \equiv g_{a ; \lambda \psi}$ at the new fixed point. In the work [9], this quantity was interpreted as ground-state degeneracy of the boundary CFT with boundary condition $a$, and it was conjectured that $g_{a}(\lambda)$ decreases along the RG trajectory; see also [1] for a perturbative proof of this "g-theorem". The formula [9]

$$
\ln \left(g_{a}(\lambda)\right)=\ln \left(g_{a}\right)-\pi^{2} y C_{\psi \psi \mathbf{1}}^{a a a} \lambda^{2}-\frac{2 \pi^{2}}{3} C_{\psi \psi \psi}^{a a a} C_{\psi \psi \mathbf{1}}^{a a a} \lambda^{3}+O\left(\lambda^{4}\right)
$$

is obtained from the general prescription (12) in a similar way as $\beta(\lambda)$; while only the OPE (more precisely the fusion channel yielding $\psi$ from $\psi \times \psi$ ) plays a role for the latter, we have to integrate two- and three-point functions of the perturbing field to arrive at $g_{a}(\lambda)$ and eq. (15). At the fixed point, the logarithm of the ground-state degeneracy is shifted from the original value by

$$
\Delta \ln \left(g_{a}\left(\lambda^{*}\right)\right)=-\frac{\pi^{2}}{3} \frac{C_{\psi \psi \mathbf{1}}^{a a a}}{\left(C_{\psi \psi \psi}^{a a a}\right)^{2}} y^{3}
$$

Due to the specific coefficients in eqs. (14) and (15), this is exact up to $O\left(y^{4}\right)$-corrections - as long as the coefficients of $\lambda^{N}$ in the terms we ignore have no higher order poles in $y$. These coefficients $I_{N}(y, \varepsilon)$ are the integrals of the $N$-point functions of the perturbing field $\psi$, and their singularities in $y$ have been studied for the $\varphi_{(1,3)}$-bulk deformation in 
[24]. The arguments used there depend only on the OPE of $\psi$ and can be applied to our situation, too. It follows that the $I_{N}(y, \varepsilon)$ are regular as $y \rightarrow 0$.

Let us now specialize to Virasoro minimal models. In view of the remarks made in the previous section, we know that the boundary CFT at the fixed point (14) can again be described by a "pure" Cardy boundary state or by an integer linear combination (7) thereof. Thus, the new ground-state degeneracy $g\left(\lambda^{*}\right)$ must be contained in (or be a sum of values in) the list (11), at least to accuracy $O\left(y^{4}\right)$. If we focus on perturbations by the least relevant boundary field $\psi=\psi_{(1,3)}$ in the boundary model $M_{m}$ with some boundary condition $\| a\rangle\rangle \neq \|(r, 1)\rangle\rangle$, this precision becomes high for large values of $m$, since $y=2 /(m+1)$.

In order to determine the new boundary condition at the fixed point $\lambda^{*}$, we first expand the ratio of two ground-state degeneracies $g_{a}, g_{b}$ from eq. (11) in powers of $1 /(m+1)$ :

$$
\begin{aligned}
\ln \left(\frac{g_{b}}{g_{a}}\right)=\ln \left(\frac{\beta_{1} \beta_{2}}{\alpha_{1} \alpha_{2}}\right)+ & \frac{\pi^{2}}{6}\left(\alpha_{1}^{2}+\alpha_{2}^{2}-\beta_{1}^{2}-\beta_{2}^{2}\right)(m+1)^{-2} \\
& +\frac{\pi^{2}}{3}\left(\alpha_{1}^{2}-\beta_{1}^{2}\right)(m+1)^{-3}+O\left((m+1)^{-4}\right)
\end{aligned}
$$

where we have used the notation $a=\left(\alpha_{1}, \alpha_{2}\right), b=\left(\beta_{1}, \beta_{2}\right)$, and where we assume that the labels for the boundary conditions are close to the origin of the conformal grid, i.e. $\alpha_{i}, \beta_{i} \ll m$. (Note that the next formula shows that if the $\alpha_{i}$ satisfy this restriction then so do the $\beta_{i}$.)

If we plug in Runkel's OPE structure constants from the Appendix into the perturbative formula (16), we obtain

$$
\Delta \ln \left(g_{a}\left(\lambda^{*}\right)\right)=-\frac{\pi^{2}}{3}\left(\alpha_{2}^{2}-1\right)(m+1)^{-3}+O\left((m+1)^{-4}\right) .
$$

Now we compare the expressions $(17)$ and (18) order by order in $1 /(m+1)$ to determine the new boundary condition $b=\left(\beta_{1}, \beta_{2}\right)$; one finds the equations

$$
\alpha_{1} \alpha_{2}=\beta_{1} \beta_{2}, \quad \alpha_{1}^{2}+\alpha_{2}^{2}=\beta_{1}^{2}+\beta_{2}^{2}, \quad \beta_{1}^{2}-\alpha_{1}^{2}=\alpha_{2}^{2}-1 .
$$

If the starting boundary condition $a=\left(\alpha_{1}, \alpha_{2}\right)$ satisfies $\alpha_{1}=1$, there is a (unique) solution over the positive integers,

$$
\beta_{1}=\alpha_{2}, \quad \beta_{2}=\alpha_{1},
$$

which corresponds to a flow from boundary condition $a=(1, r)$ to $b=(r, 1)$. For specific small values of $m$, these RG trajectories have already been found by TBA methods $[7,8]$. For $a \neq(1, r)$, one cannot find a Cardy boundary state $\| b\rangle\rangle$ with $b \in I_{\text {Kac }}$ such that eqs. (19) are fulfilled. This means that we have to pass to superpositions of Cardy boundary states as in eq. (7), and to replace $g_{b}$ in (17) by a sum $g_{\text {sup }}=g_{b_{1}}+\ldots+g_{b_{N}}$ with $g_{b_{l}}$ from eq. (11), each $b_{l}=\left(\beta_{1}^{l}, \beta_{2}^{l}\right)$ possibly occuring more than once. Then we get, after some algebra,

$$
\begin{aligned}
\ln \left(\frac{g_{\mathrm{sup}}}{g_{a}}\right)=\ln (\sigma)+\frac{\pi^{2}}{6} & \left(\alpha_{1}^{2}+\alpha_{2}^{2}-\sum_{l} \frac{s_{l}}{\sigma}\left(\left(\beta_{1}^{l}\right)^{2}+\left(\beta_{2}^{l}\right)^{2}\right)\right)(m+1)^{-2} \\
& +\frac{\pi^{2}}{3}\left(\alpha_{1}^{2}-\sum_{l} \frac{s_{l}}{\sigma}\left(\beta_{1}^{l}\right)^{2}\right)(m+1)^{-3}+O\left((m+1)^{-4}\right)
\end{aligned}
$$


where we have introduced the abbreviations $s_{l}:=\frac{\beta_{1}^{l} \beta_{2}^{l}}{\alpha_{1} \alpha_{2}}$ and $\sigma:=\sum_{l} s_{l}$. Now we can again compare with the perturbative result (18) and obtain the equations

$$
\sigma=1, \quad \sum_{l} s_{l}\left(\left(\beta_{1}^{l}\right)^{2}+\left(\beta_{2}^{l}\right)^{2}\right)=\alpha_{1}^{2}+\alpha_{2}^{2}, \quad \sum_{l} s_{l}\left(\beta_{2}^{l}\right)^{2}=1 .
$$

These enforce $\beta_{2}^{l}=1$ for all $l$, thus the RG flow triggered by the least relevant boundary field leads from a Cardy boundary condition $a$ to a superposition of $b_{l}=\left(\beta_{1}^{l}, 1\right)$. Depending on the actual values of $\alpha_{1}, \alpha_{2}$ and $m$, eqs. (21) may admit several solutions for the labels $\beta_{1}^{l}$. There is, however, the distinguished generic solution

$$
\beta_{2}^{l}=1, \quad \beta_{1}^{l}=\alpha_{1}+\alpha_{2}+1-2 l \quad \text { for } \quad l=1, \ldots, N:=\min \left(\alpha_{1}, \alpha_{2}\right) \text {. }
$$

A flow to a superposition of boundary conditions was already observed in [8] for the special case $m=4$ and $a=(2,2)$.

So far, all our calculations were based on the ground-state degeneracy. This leaves some ambiguities in the final boundary condition because of the symmetry $g_{(r, s)}=g_{(m-r, s)}=$

$g_{(r, m+1-s)}$. We can, however, repeat the procedures from above for one-point functions of other bulk fields and compute e.g. the change of $\left\langle\varphi_{(2,2)}(\zeta, \bar{\zeta})\right\rangle_{a ; \lambda \psi}$. This function picks up a sign under the transformations $a \equiv(r, s) \mapsto(m-r, s)$ or $a \mapsto(r, m+1-s)$. In fact, it is easy to see that the zeroth order in $\lambda$ already suffices to rule out $\left(m-\beta_{1}^{l}, \beta_{2}^{l}\right)$ and $\left(\beta_{1}^{l}, m+1-\beta_{2}^{l}\right)$ in favour of $\left(\beta_{1}^{l}, \beta_{2}^{l}\right)$ from eq. (22). With the help of higher order corrections, one can also exclude some of the non-generic solutions to eqs. (21).

\section{Concluding remarks}

Our perturbative analysis of relevant boundary deformations yields the following picture: The RG flow triggered by the least relevant boundary field $\psi_{(1,3)}$, starting from a Virasoro minimal model with Cardy boundary condition $a=\left(\alpha_{1}, \alpha_{2}\right), \alpha_{2}>1$, has a non-trivial fixed point with new boundary condition given by a superposition of $N:=\min \left(\alpha_{1}, \alpha_{2}\right)$ Cardy boundary states, namely

$$
a=\left(\alpha_{1}, \alpha_{2}\right) \stackrel{\psi_{(1,3)}}{\longrightarrow} \quad a_{\lambda^{*} \psi_{(1,3)}}=\sum_{l=1}^{N}\left(\alpha_{1}+\alpha_{2}+1-2 l, 1\right)
$$

For the time being, this result remains slightly conjectural in that we cannot exclude all the non-generic solutions to eqs. (21) by analytic means. In order to back up the RG flow pattern (23), one can resort to TCSA calculations which rest on an explicit diagonalization of the perturbed Hamiltonian in a finite-dimensional subspace of low-lying energy levels. This allows one to determine the spectrum $Z_{0, a_{\lambda^{*} \psi}}(q)$, from which the new boundary condition $a_{\lambda^{*} \psi}$ can be inferred by counting degeneracies of energy levels. This method is restricted to case-by-case studies, but in all examples tested so far the pattern (23) was confirmed [25], even for small $m$-values down to $m=3$. Likewise, the assumption $\alpha_{i} \ll m$, which we had to make for technical reasons, does not seem to play any role in the end. (When dropping this condition, the conformal grid symmetry makes the number $N$ of 
superimposed boundary conditions introduced in (22) ambiguous: We should then pass to $\left(m-\alpha_{1}, m+1-\alpha_{2}\right)$ if one of the entries in this labeling is smaller than those in $\left(\alpha_{1}, \alpha_{2}\right)$.) TBA and TCSA investigations of $\psi_{(1,3)}$ boundary flows reveal the existence of a second fixed point $\lambda_{-}^{*}$ besides the perturbative value $\lambda_{+}^{*}:=\lambda^{*}$ from eq. (14). The values $\lambda_{ \pm}^{*}$ have different signs and correspond to moving away from the original theory in directions $\pm \psi_{(1,3)}$.

From the works $[7,8]$ and from a large number of TCSA calculations [25], one is led to consider the following pattern: For large enough $\alpha_{i}$, the boundary condition $a_{\lambda_{-}^{*} \psi}$ reached by perturbing $a=\left(\alpha_{1}, \alpha_{2}\right)$ with $-\psi_{(1,3)}$ coincides with $a_{\lambda_{+}^{*} \psi}^{\prime}$ reached by perturbing $a^{\prime}:=$ $\left(\alpha_{1}, \alpha_{2}-1\right)$ with $+\psi_{(1,3)}$ as above, i.e. [25]

$$
a=\left(\alpha_{1}, \alpha_{2}\right) \stackrel{-\psi_{(1,3)}}{\longrightarrow} a_{\lambda_{-}^{*} \psi_{(1,3)}}=\sum_{l=1}^{N}\left(\alpha_{1}+\alpha_{2}-2 l, 1\right)
$$

with $N:=\min \left(\alpha_{1}, \alpha_{2}-1\right)$. In hindsight, it is clear that our perturbative analysis could not uncover this second class of fixed points, since $\Delta \ln \left(g\left(\lambda_{-}^{*}\right)\right)$ does not become small for large $m$.

Remarkably, in all these cases, perturbation with the least relevant boundary field already leads to a "stable" boundary condition: The spectrum at the new fixed point follows from eqs. (3) and (7),

$$
Z_{\mathrm{sup}}(q)=\sum_{l, l^{\prime}=1}^{N} n_{\mathrm{sup}}^{b_{l}} n_{\mathrm{sup}}^{b_{l^{\prime}}} Z_{b_{l} b_{l^{\prime}}}(q)
$$

and using formula (8) together with the fusion rules (6), we see that $\beta_{2}^{l}=1$ implies that no relevant boundary (condition changing) operators besides the identity are left in the spectrum. This phenomenon is to be contrasted to the chain of least relevant bulk flows considered in [10]. Formula (25) also shows that there are $N$ boundary operators of dimension zero contained in the spectrum of the superposition.

The Virasoro minimal models on the disk studied here are intimately connected to RSOS models on a cylinder, whose "spin variables" take values in an $A_{m}$ graph. Here, boundary conditions appear as additional restrictions on the spin variables in the layers near the cylinder ends $[26,16,19]$. The pattern $(23)$ suggests that, when a relevant perturbation with $\psi_{(1,3)}$ is turned on along the boundaries of the cylinder, the lattice model "breaks up" into $N^{2}$ independent sublattices, with boundary conditions $b_{l}, b_{l^{\prime}}$ as in (22). (The lattice interpretation of the Cardy boundary condition $\left(\beta_{1}^{l}, 1\right)$ is that the spins in the two outer layers are fixed to $\beta_{1}^{l}$ and $\beta_{1}^{l}+1$.) The partition function of such a subsystem is then given by the term $Z_{b_{l} b_{l^{\prime}}}(q)$ from eq. (25). As we mentioned in the introduction, Affleck found that this "splitting into subsystems" signals the presence of ordered phases along the boundary [12]. The meaning of the associated first order phase transitions within brane physics remains to be understood better.

One should also try to generalize the investigations of Section 3 to minimal models of the $N=2$ super Virasoro algebra. These occur as building blocks of phenomenologically interesting string backgrounds, namely of Gepner models. Boundary conditions in these 
superconformal theories [27] are closely related to D-branes in superstring compactifications on Calabi-Yau manifolds [28]. Statements on relevant boundary flows in such models should directly translate into statements on stability and bound state formation of the corresponding branes, extending ideas in [4,3] and the $\mathrm{SU}(2)$-findings of [14].

Acknowledgments We are grateful to W. Nahm, P. Pearce, V. Rittenberg, G. von Gehlen and J.-B. Zuber for useful discussions. We are particularly indebted to G. Watts for valuable suggestions and for making his unpublished results on the TCSA analysis of relevant boundary flows available to us. D.R. thanks the AEI Potsdam for hospitality. A.R. thanks King's College, London, for hospitality during a visit supported by the TMR European Superstring Theory Network.

\section{Appendix}

Here we give explicit expressions for specific structure constants of the boundary OPE of Virasoro minimal models. According to Runkel's work [21], they follow from the fusing matrices which describe a change of basis in the space of conformal blocks. We write $a=\left(\alpha_{1}, \alpha_{2}\right)$ for the boundary condition as before and introduce $\delta_{a}:=\alpha_{2}-\alpha_{1}$, along with some further abbreviations:

$$
\begin{aligned}
& A\left(\delta_{a}, \alpha_{2}\right):=\Gamma\left(\delta_{a}-\frac{\alpha_{2}}{m+1}\right) \Gamma\left(\delta_{a}-\frac{\alpha_{2}-1}{m+1}\right) \Gamma\left(1-\delta_{a}+\frac{\alpha_{2}}{m+1}\right), \\
& B\left(\delta_{a}, \alpha_{2}\right):=\Gamma^{2}\left(1+\delta_{a}-\frac{\alpha_{2}+1}{m+1}\right) \Gamma\left(1-\delta_{a}+\frac{\alpha_{2}-1}{m+1}\right), \\
& C\left(\delta_{a}, \alpha_{2}\right):=\Gamma^{2}\left(1+\delta_{a}-\frac{\alpha_{2}+1}{m+1}\right) \Gamma\left(-\delta_{a}+\frac{\alpha_{2}+1}{m+1}\right), \\
& D\left(\delta_{a}, \alpha_{2}\right):=\Gamma\left(1+\delta_{a}+\frac{\alpha_{2}-1}{m+1}\right) \Gamma\left(-1+\delta_{a}-\frac{\alpha_{2}-2}{m+1}\right) \Gamma\left(2-\delta_{a}+\frac{\alpha_{2}-2}{m+1}\right) .
\end{aligned}
$$

Using the recursive formula for the F-entries given in [21], the structure constants needed for the investigation of relevant perturbations with $\psi=\psi_{(1,3)}$ can be written as follows:

$$
\begin{aligned}
& C_{\psi \psi \mathbf{1}}^{a a a}= \frac{\Gamma\left(2-\frac{2}{m+1}\right) \Gamma\left(2-\frac{3}{m+1}\right) \Gamma\left(1-\frac{2}{m+1}\right)}{\Gamma\left(1-\frac{1}{m+1}\right)}\left(\frac{A\left(\delta_{a}, \alpha_{2}\right)}{B\left(\delta_{a}, \alpha_{2}\right)}+\frac{A\left(-\delta_{a},-\alpha_{2}\right)}{B\left(-\delta_{a},-\alpha_{2}\right)}\right), \\
& C_{\psi \psi \psi}^{a a a}= \frac{\Gamma^{2}\left(-1+\frac{2}{m+1}\right) \Gamma\left(-1+\frac{3}{m+1}\right) \Gamma\left(2-\frac{2}{m+1}\right) \Gamma\left(2-\frac{3}{m+1}\right)}{\Gamma\left(-2+\frac{4}{m+1}\right) \Gamma^{2}\left(\frac{1}{m+1}\right) \Gamma^{2}\left(1-\frac{1}{m+1}\right)}\left(\frac{A\left(\delta_{a}, \alpha_{2}\right)}{C\left(\delta_{a}, \alpha_{2}\right)}+\frac{A\left(-\delta_{a},-\alpha_{2}\right)}{C\left(-\delta_{a},-\alpha_{2}\right)}\right) \\
& \quad+\frac{\Gamma\left(-2+\frac{3}{m+1}\right) \Gamma\left(3-\frac{4}{m+1}\right) \Gamma\left(3-\frac{3}{m+1}\right)}{\Gamma\left(1-\frac{1}{m+1}\right) \Gamma\left(\frac{1}{m+1}\right) \Gamma\left(2-\frac{2}{m+1}\right)}\left(\frac{A\left(\delta_{a}, \alpha_{2}\right)}{D\left(\delta_{a}, \alpha_{2}\right)}+\frac{A\left(-\delta_{a},-\alpha_{2}\right)}{D\left(-\delta_{a},-\alpha_{2}\right)}\right) .
\end{aligned}
$$

Now, we can expand the ratio $C_{\psi \psi \mathbf{1}}^{a a a} /\left(C_{\psi \psi \psi}^{a a a}\right)^{2}$ in $1 /(m+1)$, with the rather simple result

$$
\frac{C_{\psi \psi \mathbf{1}}^{a a a}}{\left(C_{\psi \psi \psi}^{a a a}\right)^{2}}=\frac{1}{8}\left(\alpha_{2}^{2}-1\right)+O\left((m+1)^{-1}\right)
$$




\section{References}

[1] I. Affleck, A.W.W. Ludwig, Exact conformal-field-theory results on the multichannel Kondo effect: Single-fermion Green's function, self-energy, and resistivity, Phys. Rev. B48 (1993) 7297

[2] P. Fendley, A.W.W. Ludwig, H. Saleur, Exact conductance through point contacts in the $\nu=1 / 3$ fractional quantum Hall effect, Phys. Rev. Lett. 74 (1995) 3005, cond-mat/9408068; Exact nonequilibrium transport through point contacts in quantum wires and fractional quantum Hall devices, Phys. Rev. B52 (1995) 8934, cond-mat/9503172; Exact non-equilibrium DC shot noise in Luttinger liquids and fractional quantum Hall devices, Phys. Rev. Lett. 75 (1995) 2196, cond-mat/9505031

[3] E. Gava, K.S. Narain, M.H. Sarmadi, On the bound states of $p$ - and ( $p+2)$-branes, Nucl. Phys. B504 (1997) 214, hep-th/9704006

[4] A. Sen, SO(32) spinors of type I and other solitons on brane-antibrane pair, J. High Energy Phys. 09 (1998) 023, hep-th/9808141; Descent relations among bosonic D-branes, Int. J. Mod. Phys. A14 (1999) 4061, hep-th/9902105; Non-BPS states and branes in string theory, hep-th/9904207

[5] A. Sen, B. Zwiebach, Tachyon condensation in string field theory, hep-th/9912249

N. Berkovits, A. Sen, B. Zwiebach, Tachyon condensation in superstring field theory, hep-th/0002211

J.A. Harvey, P. Kraus, D-Branes as unstable lumps in bosonic open string field theory, hep-th/0002117

[6] J.A. Harvey, D. Kutasov, E.J. Martinec, On the relevance of tachyons, hep-th/0003101

[7] P. Fendley, H. Saleur, N.P. Warner, Exact solution of a massless scalar field with a relevant boundary interaction, Nucl. Phys. B430 (1994) 577, hep-th/9406125

P. Dorey, A. Pocklington, R. Tateo, G. Watts, TBA and TCSA with boundaries and excited states, Nucl. Phys. B525 (1998) 641, hep-th/9712197

F. Lesage, H. Saleur, P. Simonetti, Boundary flows in minimal models, Phys. Lett. B427 (1998) 85, hep-th/9802061

C. Ahn, C. Rim, Boundary flows in general coset theories, J. Phys. A32 (1999) 2509, hep-th/9805101

P. Dorey, I. Runkel, R. Tateo, G. Watts, g-function flow in perturbed boundary conformal field theories, hep-th/9909216

[8] L. Chim, Boundary S-matrix for the tricritical Ising model, Int. J. Mod. Phys. A11 (1996) 4491, hep-th/9510008

[9] I. Affleck, A.W.W. Ludwig, Universal noninteger 'groundstate degeneracy' in critical quantum systems, Phys. Rev. Lett. 67 (1991) 161

[10] A.B. Zamolodchikov, Renormalization group and perturbation theory about fixed points in twodimensional field theory, Sov. J. Nucl. Phys. 46 (1987) 1090

[11] A. Recknagel, V. Schomerus, Boundary deformation theory and moduli spaces of D-branes, Nucl. Phys. B545 (1999) 233, hep-th/9811237; Moduli spaces of D-branes in CFT-backgrounds, hepth/9903139

[12] I. Affleck, Edge critical behaviour of the 2-dimensional tri-critical Ising model, cond-mat/0005286

[13] I. Affleck, M. Oshikawa, H. Saleur, Boundary critical phenomena in the three-state Potts model, J. Phys. A31 (1998) 5827, cond-mat/9804117

[14] A.Yu. Alekseev, A. Recknagel, V. Schomerus, Brane dynamics in background fluxes and non-commutative geometry, J. High Energy Phys. 05 (2000) 010, hep-th/0003187

[15] N. Ishibashi, The boundary and crosscap states in conformal field theories, Mod. Phys. Lett. A4 (1989) 251

[16] J.L. Cardy, Boundary conditions, fusion rules and the Verlinde formula, Nucl. Phys. B324 (1989) 581

[17] D.C. Lewellen, Sewing constraints for conformal field theories on surfaces with boundaries, Nucl. Phys. B372 (1992) 654

[18] G. Pradisi, A. Sagnotti, Y.S. Stanev, Completeness conditions for boundary operators in 2d conformal field theory, Phys. Lett. B381 (1996) 97, hep-th/9603097 
[19] R.E. Behrend, P.A. Pearce, V.B. Petkova, J.-B. Zuber, Boundary conditions in rational conformal field theories, hep-th/9908036

[20] J.L. Cardy, D.C. Lewellen, Bulk and boundary operators in conformal field theory, Phys. Lett. B259 (1991) 274

[21] I. Runkel, Boundary structure constants for the A-series Virasoro minimal models, Nucl. Phys. B549 (1999) 563, hep-th/9811178; Structure constants for the D-series Virasoro minimal models, hep-th/9908046

[22] G. Felder, J. Fröhlich, J. Fuchs, C. Schweigert, The geometry of WZW branes, hep-th/9909030; Conformal boundary conditions and three-dimensional topological field theory, Phys. Rev. Lett. 84 (2000) 1659, hep-th/9909140; Correlation functions and boundary conditions in RCFT and threedimensional topology, hep-th/9912239

[23] J.L. Cardy, Conformal invariance and statistical mechanics, Lectures given at the Les Houches Summer School in Theoretical Physics, 1988

[24] A.W.W. Ludwig, J.L. Cardy, Perturbative evaluation of the conformal anomaly at new critical points with applications to random systems, Nucl. Phys. B285 (1987) 687

[25] G. Watts, unpublished results

[27] A. Recknagel, V. Schomerus, D-branes in Gepner models, Nucl. Phys. B531 (1998) 185, hepth/9712186

S. Govindarajan, T. Jayaraman, T. Sarkar, Worldsheet approaches to D-branes on supersymmetric cycles, hep-th/9907131

M. Naka, M. Nozaki, Boundary states in Gepner models, hep-th/0001037

I. Brunner, V. Schomerus, D-branes at singular curves of Calabi-Yau compactifications, hep-th/0001132

[28] H. Ooguri, Y. Oz, Z. Yin, D-branes on Calabi-Yau spaces and their mirrors, Nucl. Phys. B477 (1996) 407, hep-th/9606112

M. Gutperle, Y. Satoh, D-branes in Gepner models and supersymmetry, Nucl. Phys. B543 (1999)

73, hep-th/9808080; D0-branes in Gepner models and N=2 black holes, Nucl. Phys. B555 (1999) 477, hep-th/9902120

I. Brunner, M.R. Douglas, A. Lawrence, C. Römelsberger, D-branes on the quintic, hep-th/9906200

D.-E. Diaconescu, C. Römelsberger, D-branes and bundles on elliptic fibrations, hep-th/9910172

P. Kaste, W. Lerche, C.A. Lütken and J. Walcher, D-branes on K3-fibrations, hep-th/9912147

E. Scheidegger, D-branes on some one- and two-parameter Calabi-Yau hypersurfaces, hep-th/9912188

[26] H. Saleur, M. Bauer, On some relations between local height properties and conformal invariance, Nucl. Phys. B320 (1989) 591 